\title{
Association between serum tumor necrosis factor-alpha level and the efficacy of infliximab for refractory pouchitis after restorative proctocolectomy in patients with ulcerative colitis
}

\author{
Motoi Uchino, Hiroki Ikeuchi, Toshihiro Bando, Akihiro Hirata, Teruhiro Chohno, \\ Hirofumi Sasaki, Yuki Horio and Shiro Nakamura \\ Department of Inflammatory Bowel Disease, Hyogo College of Medicine, Hyogo, Japan
}

\begin{abstract}
:
Objectives: Although the aetiology of pouchitis after restorative proctocolectomy in ulcerative colitis (UC) remains unknown, infliximab (IFX) is often effective for this condition. However, indicators and predictors of treatment efficacy remain unclear. In this study, the association between serum tumor necrosis factor-alpha (TNF- $\alpha$ ) levels and refractory pouchitis was evaluated. Methods: We conducted a prospective study between January 2014 and April 2016. Patients with antibiotic-refractory pouchitis were treated with IFX. Serum TNF- $\alpha$ levels were measured before IFX induction. Diagnoses were confirmed using the modified Pouchitis Disease Activity Index (m-PDAI). Responders were defined as patients with an m-PDAI score lower than 5. Recurrence was defined as an m-PDAI score exceeding 5 during maintenance treatment or a need for additional treatments. Associations between serum TNF- $\alpha$ level and efficacy of IFX during 52 weeks of maintenance therapy were evaluated. Results: Thirteen patients were eligible for this study. The short-term efficacy was $8 / 13(61.5 \%)$. Four patients could not be maintained with IFX alone. The cumulative maintenance ratio was 30.8\%/52 weeks, and the cut-off value for serum TNF- $\alpha$ was $1.93 \mathrm{pg} / \mathrm{mL}$ for short-term response. Although there was no significant association between serum TNF- $\alpha$ and treatment response, IFX treatment was unsuccessful for all five patients with TNF- $\alpha$ levels below $1.93 \mathrm{pg} / \mathrm{mL}$, including four short-term non-responders and one long-term non-responder. Conclusion: Serum TNF- $\alpha$ level was not an independent predictor of IFX efficacy for refractory pouchitis. However, IFX may be effective for patients with elevated serum TNF- $\alpha$. Future studies should assess this possibility.
\end{abstract}

Keywords:

ulcerative colitis, pouchitis, tumor necrosis factor-alpha, infliximab

J Anus Rectum Colon 2017; 1(4): 106-111

\section{Introduction}

Ulcerative colitis (UC) is now thought of as a group of systemic immune diseases including colitis, gastroduodenitis, enteritis, arthritis, dermatitis, and cholangitis, among others ${ }^{1)}$. These immune disorders often develop after restorative proctocolectomy (RPC) and, like colitis, respond to immunosuppressive treatments. Among these conditions, pouchitis is the most common complication after RPC with a cumulative incidence of approximately $10 \%-50 \%{ }^{2-5)}$. Whereas most patients with pouchitis respond well to antibiotic treatment, antibiotic resistance may develop over the clinical course of disease. Consecutive treatments for refractory pouchitis, which are similar to those for colitis, include corticosteroids, immunomodulators, and anti-tumor necrosis factor-alpha (TNF- $\alpha$ ) antibodies ${ }^{6-8}$. However, only a few prospective studies with small sample sizes have evaluated the role of anti-TNF- $\alpha$ antibodies. Thus, the fundamental role of such antibodies in the treatment of refractory pouchitis remains unknown. In a prior series, although infliximab (IFX) demonstrated efficacy in treating stricture with pouchitis or fistulizing pouchitis, which are known as Crohn's-like complications, not all patients responded to IFX therapy,10). Moreover, indications for IFX in the treatment of refractory 
pouchitis remain unclear, and parameters to predict whether patients will respond to IFX remain poorly defined. Therefore, for patients with refractory pouchitis, we prospectively measured serum levels of TNF- $\alpha$ before treatment with IFX and then assessed the efficacy of this treatment with the goal of defining indications for IFX therapy.

\section{Methods}

\section{Patients}

We conducted a prospective study between January 2014 and April 2016. Patients with antibiotic-refractory pouchitis were included in this study. The characteristics of the patients, such as gender, age at surgery, duration of disease, type of disease, extent of colitis, disease severity, preoperative immunomodulating treatments, pre-operative corticosteroid dose, and existence of extra-intestinal manifestations (EIMs), were collected before surgery. Additionally, the characteristics after RPC, including the duration from ostomy closure to initial pouchitis, previous treatments for pouchitis, duration of pouchitis, and EIMs after surgery, were collected. Inflammation-related values from various blood tests, including C-reactive protein (CRP) level, white blood cell (WBC) count, erythrocyte sedimentation rate (ESR), and TNF- $\alpha$ level, were examined before IFX induction.

\section{Inclusion criteria}

Patients who were 18 years of age or older and scheduled to receive IFX treatment were included. Informed consent was obtained before induction. Refractory pouchitis was defined as frequent episodes ( $\geq$ three episodes per year) of pouchitis or persistent symptoms that necessitated long-term, continuous antibiotic therapy, including a single antibiotic (metronidazole (MNZ) or ciprofloxacin (CPFX)) for $>$ four weeks or prolonged therapy for $\geq$ four weeks with $\geq$ two antibiotics ${ }^{11}$. In this study, patients with refractory pouchitis that failed to respond to continuous treatment with MNZ, CPFX, or both, or any other immunomodulatory therapy, were assigned to IFX therapy.

\section{Diagnosis and treatment of pouchitis}

Diagnoses of pouchitis were based on clinical symptoms (increased stool frequency, bleeding, abdominal cramping, urgency, and fever) and endoscopic findings (edema, granularity, friability, loss of vascular pattern, mucous exudates, and ulceration). Additionally, the diagnosis was confirmed using the modified Pouchitis Disease Activity Index (mPDAI), a commonly used instrument for measuring disease severity ${ }^{12)}$. An m-PDAI score $\geq 5$ suggests a diagnosis of pouchitis.

The typical first-line therapy for pouchitis is oral MNZ (15 mg/kg/day) or CPFX (12 mg/kg/day) at our institution. For patients with chronic pouchitis, additional antibiotics, concomitant 5-aminosalicylate (5-ASA), or corticosteroids may be applied as second-line therapy. Other treatments, such as rifaximin, budesonide, VSL\#3 and lactulose, were either unavailable or were not sufficiently covered by insurance in Japan at the time of this study.

\section{Concurrent medications}

Concomitant administration of other immunosuppressive therapies, including corticosteroids, immunomodulators, 5ASA, or antibiotics, were not allowed within two weeks before IFX induction, even in the form of enemas or suppositories. In addition, the maintenance treatments, including antibiotics, were stopped at least two weeks before IFX induction. Moreover, such therapies were prohibited during maintenance with IFX, except if pouchitis recurred. IFX maintenance treatment was regarded as a failure if pouchitis recurred. Concomitant use of Bifidobacterium bifidum was permitted before and during maintenance with IFX.

\section{IFX treatments}

IFX induction was administered at a dose of $5 \mathrm{mg} / \mathrm{kg}$ at zero, two, and six weeks. Patients subsequently received standard maintenance therapy every eight weeks after the induction period. All patients were treated with $100 \mathrm{mg}$ of hydrocortisone and $5 \mathrm{mg}$ of d-chlorpheniramine maleate infusion to prevent infusion reactions and delayed hypersensitivity reactions. The absence of tuberculosis and other pneumonias was verified by chest computed tomography (CT) examination, tuberculin reaction (purified protein derivative test), and the interferon gamma release assay prior to IFX treatment.

\section{Exclusion criteria}

This study was limited to patients with refractory pouchitis. Patients who were suspected of having or were diagnosed with Crohn's disease by histological findings, such as epithelioid granulomas, discontinuous crypt distortion and discontinuous inflammation, and patients with transmural inflammation based on a review of colectomy specimens or endoscopic biopsy specimens were not included in this study. Patients were also excluded if they had secondary pouchitis triggered by cytomegalovirus or Clostridium difficile infection, radiation, any other form of infectious enteritis, pelvic sepsis, including an abscess or anal fistula, or obstruction due to anastomotic stricture.

\section{Evaluation and efficacy}

We evaluated the correlation between serum TNF- $\alpha$ level and efficacy of IFX treatment. The initial response was assessed at eight (range 7-9) weeks as the short-term efficacy. Clinical response evaluation was based on endoscopic and clinical data, which were included in the m-PDAI. A primary responder was defined as a patient whose m-PDAI decreased below 4 after IFX induction. Long-term efficacy was defined as a sustained response to IFX maintenance therapy alone during the 52-week treatment period. Recurrence was defined as an increase in m-PDAI over 5 points. The effi- 
Table 1. Patients Background

\begin{tabular}{lc} 
& $\mathrm{N}=13$ \\
\hline Gender (male:female) & $9: 4$ \\
Age at IFX induction for pouchitis (years) & $36.7 \pm 13.6$ \\
Pre-operative backgrounds & \\
Age at initial surgery (years) & $31.7 \pm 14.6$ \\
Duration of colitis (months) & $61.8 \pm 65.9$ \\
Severity of colitis=severe/fulminant & $5(38.5)$ \\
Pan-colitis & $13(100)$ \\
Surgical indication & $10 / 1 / 2 / 0$ \\
(refractory/perforation/TMC/cancer) & \\
Pre-operative treatments & \\
PSL use & $13(100)$ \\
Total given PSL dose (mg) & $8,981.8 \pm 8,087.0$ \\
Immunomodulators use & $6(46.2)$ \\
CNIs use & $3(23.1)$ \\
Biologics use & $4(30.8)$ \\
Post-operative backgrounds & \\
Score of mPDAI before IFX induction & $7.9 \pm 1.2$ \\
Duration from RPC (months) & $95.9 \pm 66.0$ \\
Duration from developing pouchitis (months) & $47.2 \pm 17.1$ \\
Duration from IFX induction for pouchitis & $23.7 \pm 8.8$ \\
(months) & \\
EIMs & \\
Arthritis & $2(15.4)$ \\
Erythema nodsum & $1(7.7)$ \\
Smoking habit (active smoker) & $1(7.7)$ \\
Alcohol behavior & $4(30.8)$ \\
\hline & \\
\hline & \\
&
\end{tabular}

$\mathrm{IFX}=$ infliximab, $\mathrm{TMC}=$ toxic megacolon, $\mathrm{PSL}=$ prednisolone, $\mathrm{CNI}=$ calcineurin inhibitor, $\mathrm{PDAI}=$ pouchitis disease activity index, $\mathrm{RPC}=$ restorative proctocolectomy, EIM=extra intestinal manifestation

Data are numbers with percentages in parentheses unless otherwise indicated. Continuous variables are indicated as mean \pm standard deviation.

cacy of treatment was defined based on the m-PDAI scores of the assessed parameters, which included clinical symptoms and endoscopic appearance. These parameters were assessed just prior to IFX induction and at eight to 10 weeks after the initial administration. For long-term efficacy, endoscopic examinations were occasionally performed in accordance with symptoms.

\section{Measurement of the serum TNF- $\alpha$ level}

The serum TNF- $\alpha$ level was measured just before IFX induction using a commercially available, highly sensitive enzyme-linked immunosorbent assay (ELISA) kit (R\&D Systems, Inc, Minneapolis, USA). Assays were performed exactly as recommended by the manufacturer. Fifty assays were evaluated, and the minimum detectable dose (MDD) of TNF- $\alpha$ ranged from $0.152-0.736 \mathrm{pg} / \mathrm{mL}$. The mean MDD was $0.39 \mathrm{pg} / \mathrm{mL}$. The MDD was determined by adding two standard deviations to the mean relative light units of twenty zero standard replicates and calculating the corresponding concentration.

\section{Statistical analysis}

The Wilcoxon matched pairs test was used to calculate
Table 2. Treatment for Pouchitis before Biologics Induction

\begin{tabular}{lc} 
& $\mathrm{N}=13$ \\
\hline Antibiotics & $13(100)$ \\
Topical 5-aminosalicylate & $8(61.5)$ \\
Topical corticosteroids & $5(38.5)$ \\
Topical tacrolimus & $2(15.4)$ \\
\hline
\end{tabular}

Data are numbers with percentages in parentheses unless otherwise indicated.

the significance of m-PDAI scores before and after treatment in patients with refractory pouchitis. The Mann-Whitney Utest was used to evaluate the association between the serum TNF- $\alpha$ level and response to IFX. A two-sided p-value of less than 0.05 was considered statistically significant. The Kaplan-Meier method was used for cumulative analysis. The cut-off values for each blood test were determined based on the closest left upper values in the receiver-operating characteristic (ROC) curve analysis.

\section{Ethical considerations}

All study protocols were approved by the institutional review board at Hyogo College of Medicine (No. 960). Informed consent and approval for the use of patient data were obtained before IFX induction. The study protocols were registered with the University Hospital Medical Information Network Clinical Trials Registry (UMIN-CTR 000013370).

\section{Results}

A total of 13 patients (nine males and four females) with refractory pouchitis were eligible for this study. The patient characteristics and backgrounds are shown in Table 1 . The mean ages at surgery and at IFX induction for pouchitis were $31.7 \pm 14.6$ and $36.7 \pm 13.6$ years, respectively. No patients with surgical indications of colitis-associated cancer were found.

All patients had used corticosteroids for colitis prior to surgery. Additionally, six patients were treated with thiopurine, three patients were treated with tacrolimus, and four patients were treated with anti-TNF- $\alpha$ antibodies, including three patients treated with IFX and one patient treated with adalimumab; these agents were used for colitis but did not achieve an adequate response.

EIMs were found in two patients, including a patient with both arthritis and erythema nodosum and a patient with arthritis.

Table 2 shows the previous treatments for pouchitis before IFX induction. All patients were previously treated with antibiotics. Several additional treatments also failed to achieve a cure.

S hort-term efficacy of IFX treatment was observed in 8/ 13 patients $(61.5 \%)$ (Figure 1). Of those, four patients could not be maintained with IFX during 52 weeks and required 




Figure 1. IFX efficacies in patients with refractory pouchitis. Short-term efficacy was observed in 8/13 (61.5\%) patients. Four patients were not maintained during 52 weeks of treatment. At 52 weeks, 4/13 (30.8\%) patients were not maintained with IFX alone.

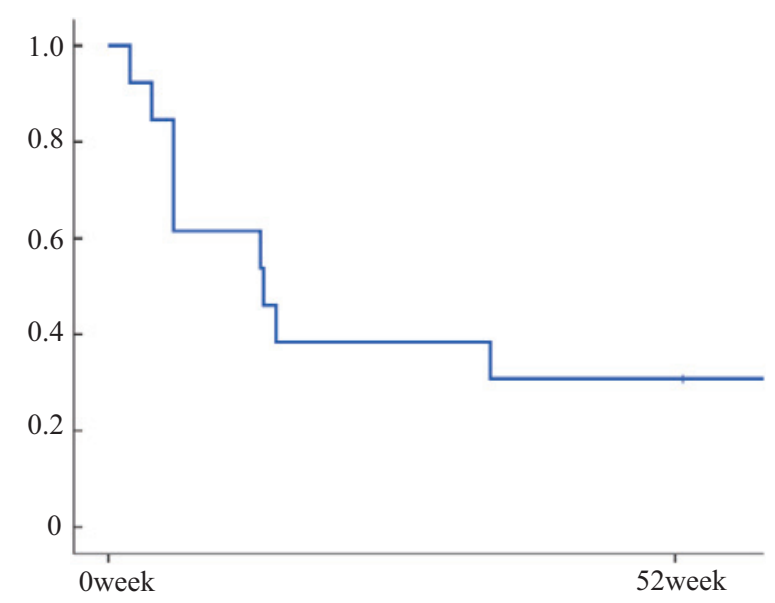

Figure 2. Cumulative maintenance rate during IFX treatment for refractory pouchitis.

The cumulative maintenance rate was 30.8\%/52 weeks; 13 patients could be maintained with IFX treatment alone.

additional treatments, including antibiotics or corticosteroids. The cumulative maintenance ratio was $30.8 \% / 52$ weeks (Figure 2).

Regarding the serum TNF- $\alpha$ concentration before IFX induction, Figure 3 presents ROC curve analysis for shortterm response to IFX and serum TNF- $\alpha$ level. The cut-off value for short-term response was $1.93 \mathrm{pg} / \mathrm{mL}$, with an area under the curve (AUC) of $0.925, \mathrm{p}<0.01$, a sensitivity of $87.5 \%$ and a specificity of $80 \%$. As shown in Figure 4, the short-term responders and non-responders had serum TNF- $\alpha$ values of $49.0 \pm 89.7$ and $1.6 \pm 0.6 \mathrm{pg} / \mathrm{mL}$, respectively, which were not significantly different $(\mathrm{p}=0.27)$. Additionally, there was no significant difference in the long-term response rate $(\mathrm{p}=0.54)$ (Figure 1). Two patients who had extremely high TNF- $\alpha$ levels (79.7 and $260 \mathrm{ng} / \mathrm{mL}$ ) had concomitant arthritis. IFX was effective for both arthritis and pouchitis during the short-term period, although this effect was not maintained over a longer duration. These patients failed to maintain within 15 weeks, which corresponded to the 4th administration of IFX. A total of five patients had TNF- $\alpha$ levels below $1.93 \mathrm{pg} / \mathrm{mL}$. Of those, four patients were nonresponders in the short-term period, and the remaining pa-

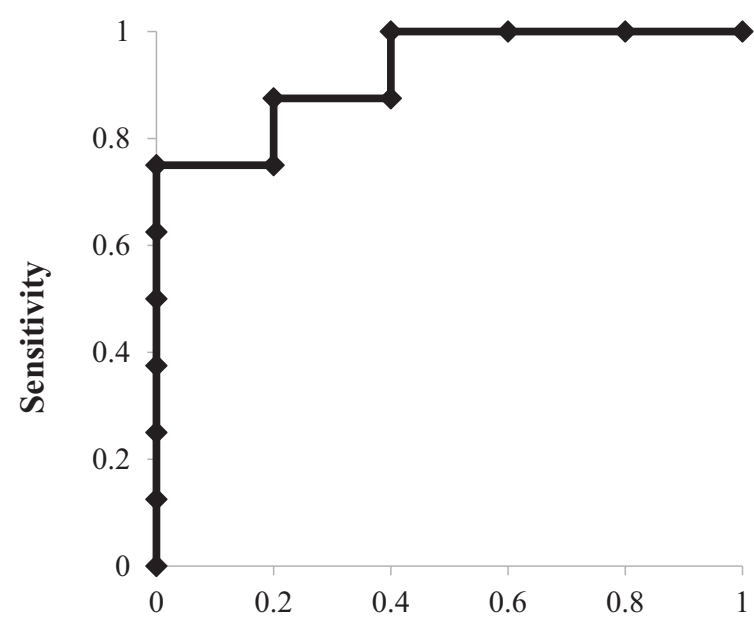

1-Specificity

Figure 3. ROC curve analysis of serum TNF- $\alpha$ level for shortterm response.

ROC curve analysis of serum TNF- $\alpha$ level showed an area under the curve (AUC) of 0.925 , with a range of 0.78 to 1.07 and $\mathrm{p}<0.01$. The cut-off value nearest to the upper left corner was $1.93 \mathrm{ng} / \mathrm{mL}$. The sensitivity and specificity were $87.5 \%$ and $80 \%$, respectively.

tient failed to maintain soon after the induction period.

Among patients treated with anti-TNF- $\alpha$ antibodies for colitis prior to RPC, all four patients with refractory pouchitis responded to IFX induction. However, only one patient who had previously used adalimumab and had a serum TNF- $\alpha$ level of $2.57 \mathrm{pg} / \mathrm{mL}$ could be maintained with 52 weeks of IFX treatment. The serum TNF- $\alpha$ levels of the remaining three patients were $2.61,260$, and $79.7 \mathrm{pg} / \mathrm{mL}$. No adverse events were observed during the study period.

With respect to the results of ROC curve analysis for short-term response to IFX and levels of other markers of inflammation, the AUC (with range in parentheses), p-value, and cut-off value were $0.74(0.40-1.08), \mathrm{p}=0.17$, and 1.0 $\mathrm{mg} / \mathrm{dL}$, respectively, for CRP; 0.69 (0.29-1.06), $\mathrm{p}=0.23$, and $19 \mathrm{~mm} / \mathrm{hr}$, respectively, for ESR; and 0.68 (0.29-1.06), p= 0.37 , and 7,610, respectively, for WBC count.

\section{Discussion}

Few large prospective trials have evaluated the efficacy of anti-TNF- $\alpha$ antibodies for pouchitis. In previous reports, the IFX response was approximately $84 \%-88 \%$ for complete or partial responses in 87 patients $^{9,13,14}$. Even in long-term follow-up periods, which included 52 weeks or a median follow-up of 21 months, the combined partial and complete response rate was $45 \%-58 \%{ }^{9,13,14)}$. The efficacy of IFX for refractory pouchitis in this study (30.8\%/1 year) and previous series $(70 \%$ for the short-term and $40 \%$ for the long-term response rate) was lower than that in other published reports ${ }^{10}$. These discrepancies may be due to differences in the study design or concomitant treatments. First, concomitant thiopurine use was not allowed in this and previous series. 


\section{Short-term}

Serum TNF- $\alpha$ $\mathrm{pg} / \mathrm{mL}$

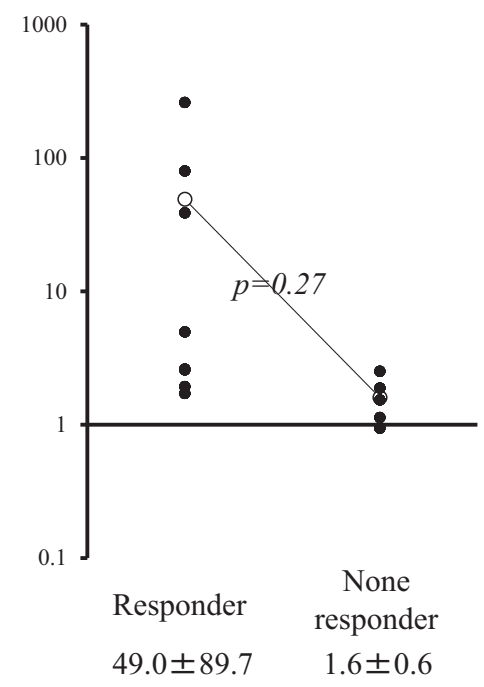

\section{Long-term}

Serum TNF- $\alpha$ $\mathrm{pg} / \mathrm{mL}$

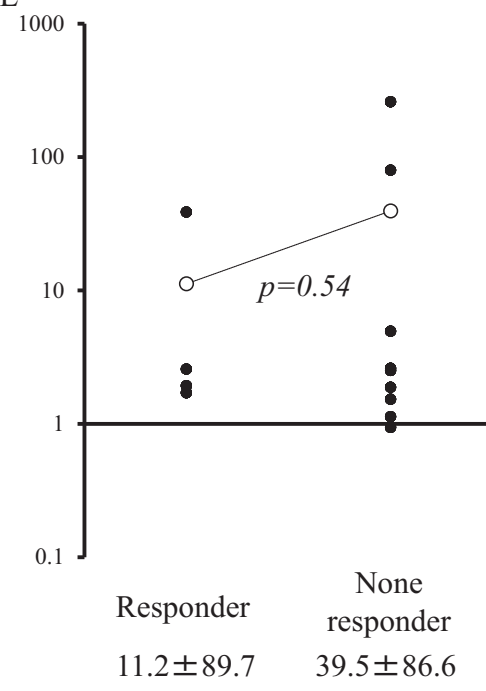

Figure 4. Comparison of the serum TNF- $\alpha$ level just before infliximab induction based on the treatment response.

In the short-term comparison, the mean \pm standard deviation of the serum TNF- $\alpha$ level was $49.0 \pm 89.7 \mathrm{ng} / \mathrm{mL}$ in responders and $1.6 \pm 0.6 \mathrm{ng} / \mathrm{mL}$ in non-responders.

In the long-term comparison, these values were $11.2 \pm 89.7 \mathrm{ng} / \mathrm{mL}$ in responders and $39.5 \pm 86.6$ $\mathrm{ng} / \mathrm{mL}$ in non-responders. There were no significant differences in these values.

Second, the patients with Crohn's-like complications were not included in this series. Third, definitions of long-term maintenance differed between studies. Thus, it is important to evaluate the similarities, differences and relationships between conventional pouchitis and pouchitis with Crohn'slike complications in further studies.

Some previous reports have demonstrated sufficient efficacy of topical steroids for the treatment of pouchitis ${ }^{15,16)}$. Budesonide is a well-tolerated treatment for refractory pouchitis with few adverse effects. Sambuelli et al. reported a good response to local budesonide among patients with pouchitis, showing an efficacy rate of $58 \%$ in a doubleblinded controlled trial of 13 cases $^{17)}$. For pouchitis, a budesonide enema may be superior to IFX treatment due to the low adverse events, low costs, and ease of application of budenoside. However, in this series and prior series, topical steroids were applied as a betamethasone enema because budesonide was not available in Japan.

The serum TNF- $\alpha$ level was not a predictive factor for the efficacy of IFX. However, 4/5 patients in the non-responder group after short-term evaluation had low TNF- $\alpha$ levels. Moreover, the effect of IFX could not be maintained over the long term for patients with extremely high TNF- $\alpha$ levels. These findings may suggest that IFX is not efficacious when serum TNF- $\alpha$ level was not elevated above cut-off level. Also, the $5 \mathrm{mg} / \mathrm{kg}$ dose of IFX may be less to have sufficient efficacy in patients with extremely high TNF- $\alpha$ levels. However, the sample size in this study was small, and such hypotheses need to be tested in larger studies. Moreover, fu- ture studies should evaluate whether patients with extraintestinal manifestations show high serum TNF- $\alpha$ levels.

Three patients treated with IFX prior to RPC responded to IFX re-induction. Although no infusion reactions were observed, none of these patients could be maintained with IFX in the long term. We measured and examined the serum TNF- $\alpha$ level just prior to IFX induction and not during maintenance therapy, as the serum TNF- $\alpha$ level could be influenced by IFX or antibodies to IFX. Moreover, we do not have precise methods for measuring the serum TNF- $\alpha$ level during IFX treatment, and independent measurement methods will need to be established in the future. Analyses that involve considering alterations in serum TNF- $\alpha$ levels and confirming the presence of anti-IFX antibodies will be required to precisely assess the examined association because decreasing responsiveness to IFX treatment could be influenced by these factors, particularly for patients previously treated with IFX before surgery who have had a long IFX withdrawal period.

One limitation of this study was that it included a small sample size at a single institution. However, we performed this study to obtain preliminary data on the association between the serum TNF- $\alpha$ level and pouchitis treatment, which can be used to inform future studies.

\section{Conclusion}

The serum TNF- $\alpha$ level does not appear to be an independent predictive factor for IFX efficacy in refractory 
pouchitis. However, IFX may be effective for patients with increased serum TNF- $\alpha$ levels, which should be evaluated in future studies.

\section{Conflicts of Interest}

There are no conflicts of interest.

\section{References}

1. Levine JS, Burakoff R. Extraintestinal manifestations of inflammatory bowel disease. Gastroenterol Hepatol (NY). 2011 Apr; 7(4): 235-41.

2. Penna C, Dozois R, Tremaine W, et al. Pouchitis after ileal pouchanal anastomosis for ulcerative colitis occurs with increased frequency in patients with associated primary sclerosing cholangitis. Gut. 1996 Feb; 38(2): 234-9.

3. Hurst RD, Molinari M, Chung TP, et al. Prospective study of the incidence, timing and treatment of pouchitis in 104 consecutive patients after restorative proctocolectomy. Arch Surg. 1996 May; 131(5): 497-500; discussion 501-2.

4. Ståhlberg D, Gullberg K, Liljeqvist L, et al. Pouchitis following pelvic pouch operation for ulcerative colitis. Incidence, cumulative risk, and risk factors. Dis Colon Rectum. 1996 Sep; 39(9): 1012-8.

5. Uchino M, Ikeuchi $H$, Matsuoka $H$, et al. Clinical features and management of pouchitis in Japanese ulcerative colitis patients. Surg Today. 2013 Sep; 43(9): 1049-57.

6. Pardi DS, Sandborn WJ. Systematic review: the management of pouchitis. Aliment Pharmacol Ther. 2006 Apr; 23(8): 1087-96.

7. Shen B. Diagnosis and treatment of patients with pouchitis. Drugs. 2003; 63(5): 453-61.

8. Calabrese C, Gionchetti P, Rizzello F, et al. Short-term treatment with infliximab in chronic refractory pouchitis and ileitis. Aliment Pharmacol Ther. 2008 May; 27(9): 759-64.
9. Ferrante M, D'Haens G, Dewit O, et al. Efficacy of infliximab in refractory pouchitis and Crohn's disease-related complications of the pouch: a Belgian case series. Inflamm Bowel Dis. 2010 Febr; 16(2): 243-9.

10. Uchino M, Ikeuchi H, Bando $\mathrm{T}$, et al. Clinical features of refractory pouchitis with penetrating lesions and the efficacy of infliximab treatment for patients with ulcerative colitis after restorative proctocolectomy. Digestion. 2015; 92(3): 147-55.

11. Heuschen UA, Allemeyer EH, Hinz G, et al. Diagnosing pouchitis: comparative validation of two scoring systems in routine followup. Dis Colon Rectum. 2002 Jun; 45(6): 776-86; discussion 786-8.

12. Shen B, Achkar JP, Connor JT, et al. Modified pouchitis disease activity index: a simplified approach to the diagnosis of pouchitis. Dis Colon Rectum. 2003 Jun; 46(6): 748-53.

13. Colombel JF, Ricart E, Loftus EV Jr, et al. Management of Crohn's disease of the ileoanal pouch with infliximab. Am J Gastroenterol. 2003 Oct; 98(10): 2239-44.

14. Barreiro-de Acosta M, García-Bosch O, Souto R, et al. Efficacy of infliximab rescue therapy in patients with chronic refractory pouchitis: a multicenter study. Inflamm Bowel Dis. 2012 May; 18 (5): 812-7.

15. Shepherd NA, Hultén L, Tytgat GN, et al. Pouchitis. Int J Colorectal Dis. 1989 Dec; 4(4): 205-29.

16. Sandborn WJ. Pouchitis following ileal pouch-anal anastomosis: definition, pathogenesis, and treatment. Gastroenterology. 1994 Dec; 107(6): 1856-60.

17. Sambuelli A, Boerr L, Negreira S, et al. Budesonide enema in pouchitis--a double-blind, double-dummy, controlled trial. Aliment Pharmacol Ther. 2002 Jan; 16(1): 27-34.

Journal of the Anus, Rectum and Colon is an Open Access article distributed under the Creative Commons Attribution-NonCommercial-NoDerivatives 4.0 International License. To view the details of this license, please visit (https://creativecommons.org/licenses/by-nc-nd/4.0/). 